\title{
Influence of Cross-sectional Shape of the Margin, Contour and Positioning of Palatal Connectors on Oral Sensation
}

\author{
Atsushi Tanaka, DDS, ${ }^{a}$ Masayuki Hideshima, DDS, PhD, ${ }^{a}$ and Takashi Ohyama, DDS, $\mathrm{PhD}^{\mathrm{b}}$ \\ ${ }^{a}$ Removable Partial Denture Prosthodontics, Department of Masticatory Function Rehabilitation, Division of \\ Oral Health Sciences, Graduate School, Tokyo Medical and Dental University, Tokyo, Japan. \\ ${ }^{\mathrm{b}}$ Tokyo Medical and Dental University, Tokyo, Japan.
}

\section{Clinical significance}

The design of bilateral dentures is such that major connectors are often placed on the palate; this impairs oral function and sensation. It was suggested that knife-edge cross-sectional shape of the margin and bilaterally symmetrical placement of a major connector would minimize the discomfort experienced in oral sensation.

\begin{abstract}
Purpose: This study assessed the influence of crosssectional shape of the margin, contour, and positioning of palatal connectors on oral sensation.

Methods: The study population comprised six healthy dentulous individuals with an average age of 26 years. Ten types of connectors were fabricated in the form of light-cured resin baseplates; four types had different cross-sectional shapes of the margins, two had different contours of the upper surface (i.e., the surface facing the oral cavity), and four varied in their placement position. The subjects' feelings when the different types of connectors were placed in their oral cavities were assessed using a questionnaire. The results were analyzed and weighted using the sigma score method, which is a tool used in the field of psychology.

Results: The results suggested that the subjects experienced greater comfort with connectors having a knife-edge-shaped margin $(P<0.05)$. Moreover, greater discomfort was reported with bilateral oblique positioning of the connector, while less discomfort was reported with bilateral symmetrical positioning $(P<$ 0.05).
\end{abstract}

Conclusion: We suggest that to achieve maximum oral comfort, palatal connectors should be constructed such that their thickness gradually increases from the margin toward the top, and they should be placed in a

\footnotetext{
Corresponding to: Dr Masayuki Hideshima

Department of Masticatory Function Rehabilitation, Division of Oral Health Sciences, Graduate School, Tokyo Medical and Dental University, Tokyo, Japan

1-5-45 Yushima, Bunkyo-ku, Tokyo 113-8549, Japan

Tel: +81-3-5803-5515, Fax: +81-3-5803-5515

E-mail: m.hideshima.rpro@tmd.ac.jp
}

Received on April 6, 2006 / Accepted on November 7, 2006 bilaterally symmetrical position.

Key words: major connector, oral sensation, sigma score method, cross-sectional shape

\section{Introduction}

Bilateral dentures for partial defects in the maxillary dentition usually incorporate a major connector to be placed across the palate, for example, palatal bars. ${ }^{1}$ A major connector stabilizes the denture and disperses the load by limiting its movement.

Regarding the mechanical properties of palatal bars, it was reported that an anteroposterior (A-P) bar was most firm, while a U-shaped palatal bar was most flexible, and that rectilinearly placed palatal bars provided maximal resistance to the load applied on the denture..$^{2}$ Furthermore, palatal bars are required to possess the property of rigidity, i.e., unbendingness. It is known that rigidity is directly proportional to the width of the bar and to the cube of the thickness. Thus, it is proved that rectilinearly placed palatal bars with an appropriate width and thickness fulfill the high rigid and firm structure requirement of dentures.

However, the placement of a major connector on the palate often impairs the oral sensation; interrupts functional tongue movements associated with speech, swallowing, and other oral functions; and results in a feeling of discomfort. ${ }^{3-5}$

Campbell $^{6}$ and other researchers ${ }^{7,8}$ studied the manner in which subjects evaluated their feelings resulting from the placement of major connectors by employing subjective assessment methods. They reported that major connectors extending between opposite maxillary molars were desirable because they covered a smaller palatal area than that covered by other major connectors. Miyake ${ }^{9}$ analyzed the sensation in 
the oral mucosa by using a two-point discrimination threshold to assess the placement position of major connectors, and they reported that a connector placed between the bilateral maxillary first molars caused a slight disorder in sensation. Sekizuka ${ }^{10}$ studied the oral stereognosis ability of the tongue by blocking the palate with a metal plate, and they recommended that major connectors should be placed posterior to the premolars. Watanabe ${ }^{11}$ showed how the position and thickness of the palatal bar affects the speech function, and stated that it was necessary to realize the manner in which the feeling of discomfort develops, when a major connector is placed.

To ensure that an individual is adapted to a particular shape of a major connector, Sato et $\mathrm{al}^{12}$ proposed a method to assess an individual's reactions to different types of connectors by using light-cured resin baseplates.

Thus, with regard to designing a palatal bar, it appears desirable from mechanical and sensorial viewpoints to place the bar rectilinearly in the palatal region between the bilateral molars. However, there have been no detailed studies on either the cross-sectional shape of the palatal connectors, particularly that of the margins and the upper surface contour, or the bilateral symmetry of placement. Therefore, in the present study, we sought to determine the sensorially optimal cross-sectional shape and position of palatal connectors by comparing a variety of major connectors.

\section{Materials and methods}

\section{Subjects}

Six male subjects aged 24-28 years (average: 26 years) with no abnormalities in the oral function were selected. The length of their bilateral maxillary first molars ranged from 32.2 to $39.3 \mathrm{~mm}$ (average: $36.1 \mathrm{~mm}$, SD: 2.70), their palatal depth ranged from 17.8 to $13.4 \mathrm{~mm}$ (average: $15.3 \mathrm{~mm}$, SD: 1.71), and the length:depth ratio ranged from 2.74 to 2.10 (average: 2.36, SD: 0.28). Since the shape of major connectors varies with the depth of the palate and bilateral symmetry of dentition, we tried to select subjects with similar palatal shapes. The experimental protocol was approved by the Ethics Committee of the Tokyo Medical and Dental University (No. 171).

\section{Experimental procedures}

Baseplates with experimental major connectors were fabricated for the subjects, who then assessed various factors related to the crosssectional shape of the margin, the contour of the upper surface of the connector, and the positioning of the connectors.

\section{Experimental major connectors}

A variety of experimental major connectors were fabricated in the form of light-cured resin baseplates (BASEPLATE LC, MOLTEN MEDICAL Co., Japan) on casts made from alginate impressions of the subject's maxillary arch.

\section{Cross-sectional shape of margins}

Major connectors with four different cross-sectional shapes of the margin were fabricated: Kolben (KO) type, knife-edge (KN) type, half-round (HR) type, and chamfer $(\mathrm{CH})$ type. The crosssectional shape of the margin was round in the $\mathrm{KO}$ type, acutely angled $\left(20^{\circ}\right)$ in the $\mathrm{KN}$ type, a quarter circular arc in the HR type, and acutely angled (although the angle was larger $\left(35^{\circ}\right)$ than that in $\mathrm{KN}$ ) and moderately curvilinear in the CH type (Fig. 1).

These major connectors were constructed such that the midline of the connector conformed to a straight line connecting the midpoints of the bilateral maxillary first molars. The lateral part of the connector was retained by the resin baseplate, which was aligned upright in the sagittal plane passing through the bilateral canines. The resin baseplate covered the region extending from the maxillary first premolar to the second molar, and it was fabricated as thin as possible; its width was $10 \mathrm{~mm}$, and the thickness was 2 $\mathrm{mm}$ at the thickest part.

\section{Contour of the upper surface of the connector} We fabricated the following two types of major connectors with different cross-sectional contours of the upper surface, while the thickness and shape of the margin were kept constant: the peaked (PK) type with a convex contour and the flat (FL) type with a flat contour.

The connector's position while assessing the contour of the upper surface of the connector was similar to that when the cross-sectional shape of the margin was assessed, but its marginal angle was set at $35^{\circ}$. The width of the connector was 6 $\mathrm{mm}$ for the PK type and $10 \mathrm{~mm}$ for the FL type, and the thickness was $2 \mathrm{~mm}$ at the thickest por- 


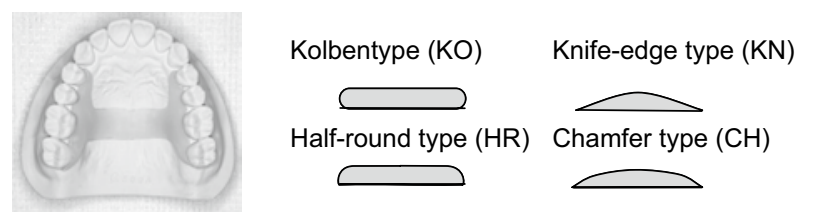

cross-sectional shape of margins

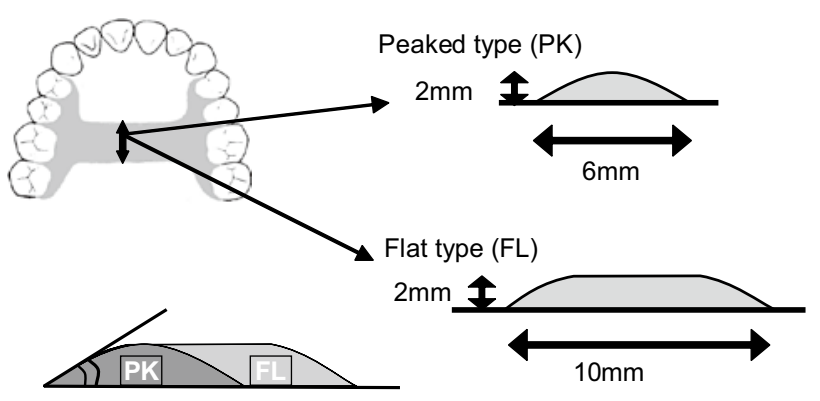

Form of the top of the connector
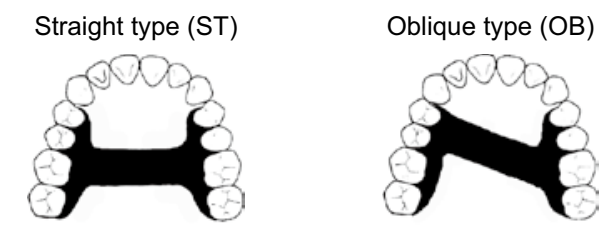

Anterior curving type (CA)

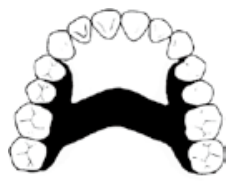

Posterior curving type (CP)

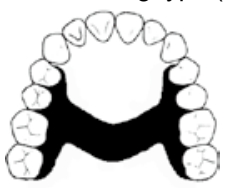

Positioning of connectors

Fig. 1 Experimental major connector. The upper figure shows the four types of cross-sectional shapes of the margins of connectors, the middle figure shows the two types of upper surface contours, and the lower figure shows the four different positioning of the connectors.

tion for both the types (Fig. 1).

\section{Positioning of connectors}

Four types of placement positions were set: the straight (ST) type, the oblique (OB) type, the anterior curving (CA) type, and the posterior curving (CP) type. In the ST type, the connector was positioned such that the midline of the connector conformed to a straight line connecting the midpoints of the bilateral maxillary first molars. In the OB type, the second premolar on the right side was rectilinearly connected to the interdental space between the first and second molars on the left side. In the CA type, the inflection point was set at the midpoint of a straight line connecting the mesial surfaces of the bilateral maxillary first molars, while in the CP type, it was set at the midpoint of a straight line connecting the distal surfaces of the bilateral maxillary first molars (Fig. 1).

\section{Questionnaire method}

The questionnaire incorporated a comparative assessment scale that evaluated paired comparisons by using an interval scale. After two different major connectors were placed in the subject's oral cavity in succession, the subject was asked to identify the connector that resulted in a stronger feeling of discomfort. Six pairs of connectors were compared for assessing the cross-sectional shape of the margins ( $\mathrm{KO}-\mathrm{KN}, \mathrm{KO}-\mathrm{HR}, \mathrm{KO}-\mathrm{CH}$, $\mathrm{KN}-\mathrm{HR}, \mathrm{KN}-\mathrm{CH}$, and $\mathrm{HR}-\mathrm{CH}$ ); the pair of $\mathrm{PK}-$ FL connectors was compared for assessing the contour of the connector's upper surface; and six pairs were compared for assessing the positioning of the connectors (ST-OB, ST-CA, ST-CP, OB-CA, OB-CP, and CA-CP). By using a table of random numbers, the connectors were placed in a random order by an examiner. In addition, to avoid any visual bias, the examiner placed the connectors directly in the oral cavity of the subjects who were instructed to close their eyes. Thereafter, the subjects were asked to select one of the five alternatives provided for each questionnaire item. For example, when the subjects compared connectors $\mathrm{A}$ and $\mathrm{B}$, they selected an answer from the following options: definitely A, prefer A, no difference between A and B, prefer B, or definitely $\mathrm{B}$.

To assess the subjects' feelings about the crosssectional shape of the margin and the contour of the connector's upper surface, the questionnaire expected responses related to six aspects-step, thickness, feeling of narrowed oral cavity, feeling of discomfort at rest, while speaking, and while swallowing (Table 1). To assess the step, the subjects were asked to feel the margins of the connectors with their tongue, and identify the particular connector out of each pair in which they felt the step. For thickness, the subjects were asked to indicate the connector, out of the pair, that felt thicker to their tongue. To assess the feeling of narrowed oral cavity, we asked about the feeling of narrowness of the oral cavity during the placement of the connectors. The feeling of discomfort at rest was assessed during slight lip-closing. For checking the speech function, we instructed the subjects to pronounce the test words-"yakin," "ashika," "ahiru," and "arika" that contain the syllables "ki," "shi," "hi," and "ri"; it is generally considered that the pronunciations of these syl- 
Table 1 Questionnaire for each cross-sectional shape of the margins and the five alternatives provided.

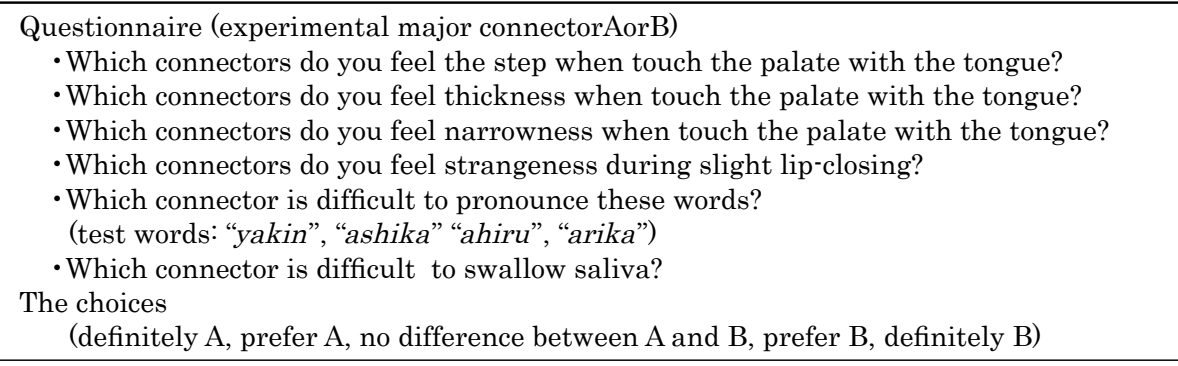



lables are easily disturbed due to the placement of a connector on the palate. ${ }^{13}$ We asked the subjects whether they found it difficult to pronounce these words. To assess swallowing, the subjects were asked to note any difficulty in swallowing saliva.

Connector's with the same cross-sectional shape of the margin were used for comparing different connector positioning. Therefore, we deleted the items of step and thickness from the questionnaire described above and added an item that evaluated the feeling of discomfort when the tongue actively touched the connectors. This was used as the questionnaire to assess connector positioning, and it thus comprised four items-feeling of discomfort at rest and feeling of discomfort while touching the connector with the tongue, speaking, and swallowing.

\section{Analytical method}

After the subjects completed the questionnaire, the ratio of each selected answer was determined. Based on these results, sigma scores were calculated. The sigma score method, which is used in the field of psychology, converts the ordinal scale of a particular category to an interval scale so that data can be expressed as a score (Fig. 2). ${ }^{14-16}$ The ratio of the selected answer to each category (definitely, prefer, no difference) is regarded as the ratio of the area under the normal distribution curve, where the mean $=0$ and the standard deviation $=1$, and the ratio of the selected answer is converted into a score that is calculated from the vertical coordinate values of the curve corresponding to the ratio. In other words, after the questionnaire's answers are obtained, the total number of selected categories (definitely, prefer, no difference) in each questionnaire item is added up and is used to calculate the ratio of each selected answer. Next, the vertical coordinate values of the normal distribution curve corresponding to the ratio of each selected answer are calculated. Using these coordinate values, the sigma score is calculated following the procedure shown in Figure 2. Thus, it becomes possible to calculate and weigh a score that corresponds to the ratio of each selected answer. Using these sigma scores, we compiled the results of the questionnaire.

The feeling of discomfort, as reported in the 
Table 2 Sigma scores for each cross-sectional shape of the margins and each type of upper surface contour (upper table) and each positioning of the connector (lower table).

Cross-sectional shape

\begin{tabular}{|c|c|c|c|c|}
\hline & Ratio of answers & $\sigma$ & Conversion score & Easy-to-use score \\
\hline Prefer & 0.389 & 0.9858 & 2.1583 & 2.1 \\
\hline Definitely & 0.317 & -0.1230 & 1.0495 & 1 \\
\hline No difference & 0.294 & -1.1725 & 0.0000 & 0 \\
\hline \multicolumn{5}{|c|}{ Positioning connectors } \\
\hline & Ratio of answers & $\sigma$ & Conversion score & Easy-to-use score \\
\hline Definitely & 0.547 & 0.7248 & 2.1339 & 2.1 \\
\hline Prefer & 0.257 & -0.4639 & 0.9452 & 0.9 \\
\hline No difference & 0.197 & -1.4092 & 0.0000 & 0 \\
\hline
\end{tabular}

questionnaire of the present study, was ranked unidirectionally toward the positive direction, and higher scores represented stronger feelings of discomfort.

For statistical analysis, we used statistical software SPSS version 10J (SPSS Japan Inc, Tokyo, Japan). Respective major connectors were compared using one-way analysis of variance and multiple comparisons (Scheffe's test). The level of significance was set at $P<0.05$.

\section{Results}

\section{Sigma scores of answers to questionnaire items (Table 2)}

The total numbers of the selected answers for each questionnaire item were determined, and the sigma scores were calculated. Since the questionnaire items for both cross-sectional shape of the margin and the contour of the upper surface of the connector were the same, the sigma values were calculated after combining the scores. The ratios of the selected answers were 0.317 for "definitely," 0.389 for "prefer," and 0.294 for "no difference"; the calculated sigma values were $-0.123,0.985$, and -1.172 , respectively. The sigma score for "no difference" was the smallest, and it was converted to an easy-to-use value of 0 . Thus, the values for "definitely," "prefer," and "no difference" were converted to $1.04,2.20$, and 0 , respectively.

In the experiment to assess the positioning of the connectors, the ratios of the selected answers were 0.547 for "definitely," 0.257 for "prefer," and 0.197 for "no difference"; the calculated sigma values were $0.724,-0.463$, and -1.409 , respectively. The values for "definitely," "prefer," and "no difference" were converted to $1.04,2.20$, and 0 , respectively, as described above.

\section{Analysis of results \\ Cross-sectional shape of margin}

The comparison of the scores of the four types of margins yielded the following values: 7.6 for the $\mathrm{KO}$ type, 6.7 for the HR type, 4.1 for the $\mathrm{CH}$ type, and 3.1 for the $\mathrm{KN}$ type. Thus, the scores were ranked in a descending order from $\mathrm{KO}, \mathrm{HR}$, $\mathrm{CH}$, to $\mathrm{KN}$ type. The score of the $\mathrm{KN}$ type was significantly smaller than that of either KO or HR types $(P<0.05)$ (Fig. 3).

\section{Contour of the upper surface}

The comparison of the scores of the two types of the upper surface contours yielded the following values: 2.3 for the PK type and 1.9 for the FL type. Thus, the scores were ranked in a descending order from PK to FL; however, there was no significant difference between these two types $(P<0.05)$.

\section{Positioning of the connectors}

The comparison of the scores of the four different types of connector positioning yielded the following values: 6.2 for the ST type, 11.9 for the OB type, 3.2 for the CA type, and 5.4 for the CP type. Thus, the scores were ranked in a descending order from OB, PP, CP, to CA types. The OB type showed a significantly higher score when compared with the scores of the other connectors $(P<0.05)$, and the score of the CP type was significantly higher than that of the CA type $(P<0.05)$ (Fig. 4). 


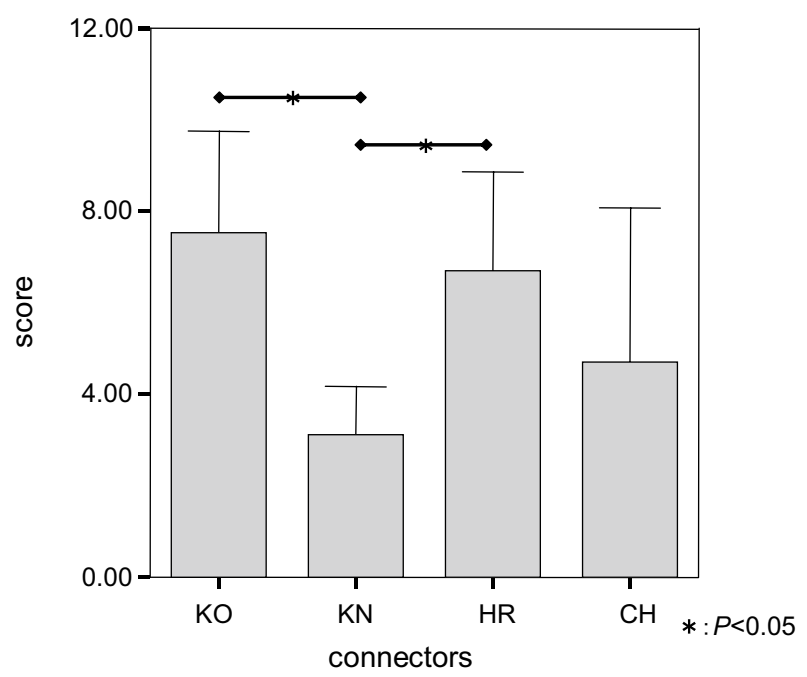

Fig. 3 Comparison of the scores for each cross-sectional shape of the margin.

\section{Discussion}

As an experimental major connector, we used light-cured resin baseplates, which were simple to fabricate and fit, and their use enabled us to achieve uniform thickness and improved accuracy. In the present study, we aimed to simulate the shape of major connectors that are made using both metal and resin. The thickness of the resin base was $2 \mathrm{~mm}$, which is approximately the same as that used in clinical procedures. Although the thickness of the resin base was greater than that of a metal connector, we considered that it was appropriate in distinguishing differences in shape and testing the differences in sensation. According to Aridome et $\mathrm{al}^{17}$, the average width of a major connector designed by each of the highly experienced doctors was about $10 \mathrm{~mm}$; hence, a width of $10 \mathrm{~mm}$ and a thickness of $2 \mathrm{~mm}$ were selected for this study.

In this study, we selected palates with a normal shape. Since an abnormal palatal shape such as that due to an existing torus palatinus or a constricted arch would unfavorably affect the oral sensation.

Studies involving the measurement of subjective items or "attitudes," such as the present study, typically use psychological methods of measurement; however, psychological language cannot be used to describe these items. It therefore appears important to find a method to translate these results into a quantitative form. ${ }^{15,16}$

In the assessment of sensation, we generally use the visual analog scale (VAS) method or a

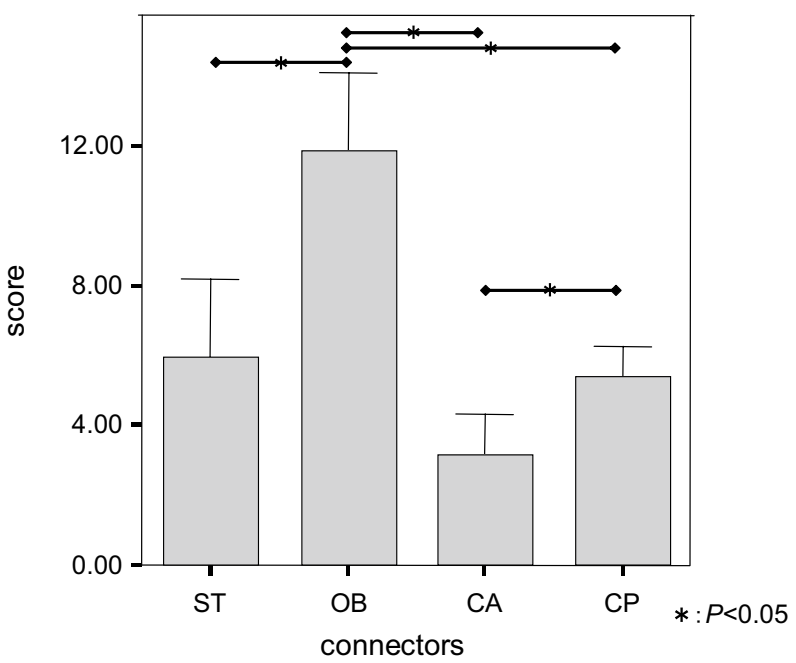

Fig. 4 Comparison of scores for each positioning of the connectors.

simplified scale employing the code number of the category as the score. The sigma score method employed in the present study was developed by Likert and is utilized in the field of psychology. ${ }^{14}$ This method converts the ordinal scale of a particular category to an interval scale so that it can be expressed as a score. Generally, the differences between the categories are expressed using the same interval, e.g., "definitely"=1, "prefer" $=0.5$, and "no difference" $=0$. Moreover, by using this method, it is possible to obtain a score proportional to the ratio of the selected answer. According to Likert, the correlation coefficient between the simplified scale score and the sigma score is equal to or higher than 0.99 , indicating that there is no difference in the results between the sigma score method and the more commonly used method. ${ }^{14,16}$ In the present study, although the comparison of the results obtained by the simplified method and those obtained by the sigma score method showed some differences in the scores themselves, there was no difference in the subjects' assessment of the rank order of the connectors. We therefore decided that the sigma score method would be useful in this study because it would not only yield almost the same results as those obtained by conventional methods but also allow the weighting of the results.

Concerning the analytical method, a number of different methods are used to compare various types of connectors. One method involves judgment based on rank correlation after assessing each connector individually. For example, to evaluate the sensations brought about by the use 
of different connectors, Campbell ${ }^{6}$ placed each connector in the subjects' oral cavities for one day and had them assess each connector. In the rank correlation of all the connectors and the comparison of connectors on different days, the results appeared to be affected by the order of placement and the selection of connectors to be compared. Accordingly, in this study, we endeavored to simplify precise judgments by limiting the comparison between a pair of connectors and by using a relative rating scale.

In this study, the score of the KN type of margin yielded significantly smaller scores than that of either the KO or HR types. The sharp margin appeared to evoke pain and feelings of discomfort readily when touched by the tongue, and it was revealed that a margin with a well-fitting smooth margin evoked less discomfort.

Comparison between the PK and FL types of connectors revealed no significant difference. We assume that this was due to the difficulty in making relative comparisons; since these two types had different widths, the conditions were not identical. Hence, to assess how the differences in the contour of the connector's upper surface would yield differences in the oral sensation, we studied the characteristics of the two types of connectors by comparing the answers selected for each questionnaire item. With regard to the questionnaire items on swallowing, speech, and feeling of narrowed oral cavity, the FL type yielded higher scores. We assumed that this was due to the fact that the proportion of intraoral volume occupied by the FL type was inevitably larger because its width was greater and its thickness was the same as that of the PK type. With regard to the feeling of discomfort at rest and the thickness, the PK type showed higher values. This may be because sharp objects in the oral cavity readily result in feelings of discomfort. However, the results obtained in this study are inadequate; therefore, more studies that include the elements of width, thickness, and the contour of the upper surface are required.

Regarding the experiment of comparing the positioning of the connectors, since the $\mathrm{KN}$ type of margin in the pilot study showed smaller scores than any other type of margin, we selected $\mathrm{KN}$ as the cross-sectional shape of the margin.

The OB type of connector showed higher scores when compared with that of the other types of connectors. In contrast to the bilaterally symmetrical ST, CA, and CP types, the OB type is bilat- erally asymmetrical. Therefore, we suggest that bilaterally symmetrical shapes lead to weaker feelings of discomfort. During oral functions such as speaking and swallowing, the tongue generally makes bilaterally symmetrical movements. Accordingly, the presence of a bilaterally asymmetrical object on the palate will cause bilaterally asymmetrical tongue contact, and this will lead to feelings of discomfort. The feeling of discomfort with the CA type was weaker than that with the CP type, probably because the shape of the tongue when it touches the palate resembled that of the CA type of connector. Future research should investigate the feeling of discomfort considering the degree of contact between the tongue and the palatal connector.

When a metal palatal connector is used, its thickness can be reduced, and this would favor oral sensation. However, in the case of resin palatal connectors, greater thickness is required to achieve adequate strength. It was suggested that the amount of discomfort would be least when connectors with the most appropriate cross-sectional margin shape and upper surface contour are used.

Limitations on this study were that the study population comprised young people with sound dentition. Ideally, elderly denture users with partially defective dentition should preferably be enrolled. However, in such individuals, the shape of the denture being used at the time of study might be another factor affecting the result of these experiments.

\section{Conclusion}

1. It was suggested the use of a connector with a cross-sectional shape of the margin designed such that the thickness increases gradually from the margin as in the $\mathrm{KN}$ type, resulted in less discomfort.

2 . With regard to the contour of the connector's upper surface, there was no significant difference between the PK and FL types of connectors when the thickness and the shape of the margin were constant.

3. It was suggested that the use of bilaterally asymmetrical connectors resulted in more discomfort than the use of bilaterally symmetrical connectors.

Based on these conclusions, it was suggested that major connectors could be designed with 
greater effectiveness from the standpoint of an individual's oral sensation.

Acknowledgements: A part of this study was presented at the $33^{\text {th }}$ Scientific Meeting of Japanese Society of Stomatognathic Function (October 13, 2004, Osaka).

This study was supported in part by a Grant-in-Aid for Scientific Research (No. 13307056) from the Ministry of Education, Culture, Sports, Science and Technology, Japan.

\section{References}

1. McGivney GP, Castleberry DJ. McCracken's removable partial prosthodontics, 10th ed. 22-58, St. Louis: C. V. Mosby, 2000.

2. Ben-Ur Z, Mijiritsky E, Gorfil C et al. Stiffness of different designs and cross-sections of maxillary and mandibular major connectors of removable partial dentures. J Prosthet Dent 81: 526-532, 1999.

3. Matsuda K. The influence of palatal plate thickness on masticatory function and phonetics. $\mathrm{J} \mathrm{Hi}$ roshima Univ Dent Soc 18: 54-71, 1986. (in Japanese)

4. Tsuga K, Nakahara Y, Shitozawa S et al. The influence of palatal plate on functional mandibular movement. J Hiroshima Univ Dent Soc 18: 351-355, 1986. (in Japanese)

5. Fujii H. Influence of masticatory movements of tongue on the food pulverization. Jap J Oral Biol 13: 485-498, 1971. (in Japanese)

6. Campbell LD. Subjective reactions to major con- nector designs for removable partial denture. J Prosthet Dent 37: 507-516, 1977.

7. LaVere AM, Krol AJ. Selection of major connector for the extension-base removable partial denture. J Prosthet Dent 30: 102-105, 1973.

8. Wagner AG, Traweek FC. Comparison of major connectors for removable partial dentures. J Prosthet Dent 47: 242-245, 1982.

9. Miyake N. Two-point discrimination thresholds in palatal mucosa. The Sikagakuho 52: 125-128, 1952. (in Japanese)

10. Sekizuka Y. Experimental studies on the influence of upper-denture planning on the sensation of presence of a foreign body in tongue. The $\mathrm{Si}^{-}$ kagakuho 73: 1044-1066, 1973. (in Japanese)

11. Watanabe M. Influence of major connector on speech (analysis of speech recognition using the pattern matching). Ohu Univ Dent J 27: 151-161, 2000 .

12. Sato Y, Yuasa Y, Ukai T et al. Trial patterns for selection of major connector. Int J Prosthodont 3: 175-178, 1990.

13. Hirota J. The phonetics consideration for denture design. The Sikagakuho 58: 1-6, 1958. (in Japanese)

14. Likert RA. Technique for the measurement of attitudes. Arc Psychol 140: 1-55, 1932.

15. Yoshida M. Psychological statistics, 132-138, Tokyo: Maruzen, 1976. (in Japanese)

16. Ohyama T. Measurement of psychology and statistics, 213-216, Tokyo: Yuhikaku, 1982. (in Japanese)

17. Aridome K, Yamazaki M, Baba K et al. Bending properties of strengthened Ti-6Al-7Nb alloy major connectors compared to $\mathrm{Co}^{-} \mathrm{Cr}$ alloy major connectors. J Prosthet Dent 93: 267-273, 2005. 